Proceedings of the

International Geometry Center

Vol. 14, no. 1 (2021) pp. 1-18

\title{
On inverse problem for tree of Stieltjes strings
}

\author{
Anastasia Dudko, Vyacheslav Pivovarchik
}

\begin{abstract}
For a given metric tree and two strictly interlacing sequences of numbers there exits a distribution of point masses on the edges (which are Stieltjes strings) such that one of the sequences is the spectrum of the spectral problem with the Neumann condition at the root of the tree while the second sequence is the spectrum of the spectral problem with the Dirichlet condition at the root.
\end{abstract}

Анотація. Для заданого метричного дерева та двох послідовностей чисел, які чергуються, існує розподіл точкових мас на ребрах (котрі є стільтьєсівськими струнами) такий, що одна з послідовностей $є$ спектром спектральної задачі з умовою Діріхле у корені, тоді як друга послідовність $є$ спектром задачі з умовою Неймана у корені.

\section{INTRODUCTION}

Finite-dimensional spectral problems on an interval were considered in [5] (some recent results see in $[16,17]$ and applications in $[1,4]$ ). Finitedimensional spectral problems on graphs occur in various fields of physics: mechanics of transverse vibrations of strings [6-8], longitudinal vibrations of point masses joined by springs [11], synthesis of electrical circuits [3,9]. By inverse problem we mean recovering the parameters of the problem using the spectra of system vibrations. Such a problem on an interval was completely solved in [5], see [18] for generalization. For a star graph the inverse problem was solved in $[2,14,15,19]$. In these papers conditions on sequences of numbers where obtained necessary and sufficient to be the

2010 Mathematics Subject Classification: 34A55, 39A70, 70J30, 70F17

Keywords: Neumann boundary condition, Dirichlet boundary condition, balance of forces condition, eigenvalues, spectrum, Stieltjes function

Ключові слова: гранична умова Неймана, гранична умова Діріхле, умова балансу сил, власні значення, спектр, функція Стілтьєса

DOI: http://dx.doi.org/10.15673/tmgc.v14i1.1783 
spectra of spectral problems on the whole graph and on the edges of it. It was shown that the solution of this inverse problem is unique only in the case of simple spectra.

Spectral problems on trees were considered in [12] where it was assumed that the interior vertices of the tree are free of point masses. In this paper we consider the case where point masses can be presented at the interior vertices.

In Section 2 we describe the spectral problems generated by Stieltjes string equations on a tree with Dirichlet and Neumann conditions at the root which is any of pendant vertices.

Section 3 contains some auxiliary results on Stieltjes functions.

In Section 4 we solve the direct problem, i.e. we show that the quotient of characteristic polynomials of the Dirichlet and Neumann problems can be expanded into branching continued fraction.

In Section 5 we solve the inverse problem of recovering values of point masses and the lengths of subintervals between them using the total lengths of the edges, the spectra of two problems: with the Dirichlet condition at the root, first, and the Neumann condition, the second (Theorem 5.1). Also we consider the inverse problem of recovering values of point masses and the lengths of subintervals between them using the numbers of masses on the edges, the spectra of two problems: with the Dirichlet condition at the root, first, and the Neumann condition, the second (Theorem 5.2).

\section{Statement of Spectral PRoblems}

Let $T$ be a plane metric tree with $q \geqslant 2$ edges. We denote by $v_{i}$ the vertices, by $d\left(v_{i}\right)$ their degrees, by $e_{j}$ the edges, by $l_{j}$ their lengths by $n_{j} \geqslant 0$ the numbers of point masses $m_{1}^{(j)}, m_{2}^{(j)}, \ldots, m_{n_{j}}^{(j)}$ which divide the string into the subintervals $l_{0}^{(j)}, l_{1}^{(j)}, \ldots, l_{n_{j}}^{(j)}\left(l_{k}^{(j)}>0\right.$ for $j=0,1, n_{j}-1$, $\left.l_{n_{j}}^{(j)} \geqslant 0, m_{k}^{(j)}>0, l_{j}=\sum_{k=0}^{n_{j}} l_{k}^{(j)}\right)$. The case of $l_{n_{j}}^{(j)}=0$ corresponds to location of $m_{n_{j}}^{(j)}$ at an interior vertex. An arbitrary vertex is chosen to be the root. The pendant vertices are free of masses. All the edges we direct away from the root.

The root $\mathbf{v}$ is the beginning of a subinterval of length $l_{0}^{(j)}$ on each edge $e_{j}$ incident with the root. Each other vertex $v_{i}$ have one incoming edge $e_{j}$ ending with a subinterval of the length $l_{n_{j}}^{(j)}$, while each outgoing edge $e_{r}$ begins at $v_{i}$ with an interval of lengths $l_{0}^{(r)}$.

The degree of a vertex $v_{i}$ is denoted by $d\left(v_{i}\right)$, the indegree of it by $d^{+}\left(v_{i}\right)$ while the outdegree by $d^{-}\left(v_{i}\right)$. It is clear that $d^{+}\left(v_{i}\right)=1$ for each $v_{i} \neq \mathbf{v}$ 
and $d^{+}(\mathbf{v})=0$. At each pendant vertex $v_{i}$ which is not the root we have $d^{-}\left(v_{i}\right)=0$.

It is assumed that the tree is stretched and the pendant vertices except the root are fixed. We consider two cases: in the first the root is fixed (Dirichlet problem) while in the second the root is free to move in the direction orthogonal to the equilibrium position of the tree (Neumann problem). The tree can vibrate in the direction orthogonal to the equilibrium position of the strings. The transverse displacement of the mass $m_{k}^{(j)}$ we denote by $w_{k}^{(j)}(t)$, the displacement of the root by $\mathbf{w}(t)$ where $t$ is time.

If an edge $e_{j}$ is incoming for an interior vertex $v_{i}$ then the displacement of the incoming end of the edge is denoted by $w_{n_{j}+1}^{(j)}(t)$, while if an edge $e_{r}$ is outgoing for a vertex $v_{i}$ then the displacement of the outgoing end of the edge is denoted by $w_{0}^{(r)}(t)$. Using such notation vibrations of the tree can be described by the system of equations

$$
\begin{gathered}
\frac{w_{k}^{(j)}(t)-w_{k+1}^{(j)}(t)}{l_{k}^{(j)}}+\frac{w_{k}^{(j)}(t)-w_{k-1}^{(j)}(t)}{l_{k-1}^{(j)}}+m_{k}^{(j)} \frac{\partial^{2} w_{k}^{(j)}}{\partial t^{2}}(t)=0, \\
\left(k=1,2, \ldots, \tilde{n}_{j} ; \quad j=1,2, \ldots, q\right),
\end{gathered}
$$

where $\tilde{n}_{j}=n_{j}-1$ if $l_{n_{j}}^{(j)}=0$ and $\tilde{n}_{j}=n_{j}$ if $l_{n_{j}}^{(j)}>0$.

For each interior vertex $v_{i}$ (except of the root) with incoming edge $e_{j}$ and outgoing edges $e_{r}$ we impose the continuity conditions

$$
w_{0}^{(r)}(t)=w_{\tilde{n}_{j+1}}^{(j)}(t)
$$

for all $r$ corresponding to outgoing edges. Balance of forces at such a vertex $v_{i}$ implies

$$
\frac{w_{\tilde{n}_{j}+1}^{(j)}(t)-w_{\tilde{n}_{j}}^{(j)}(t)}{l_{\tilde{n}_{j}}^{(j)}}+\sum_{r} \frac{w_{0}^{(r)}(t)-w_{1}^{(r)}(t)}{l_{0}^{(r)}}= \begin{cases}0, & \text { if } l_{n_{j}}^{(j)}>0 \\ m_{n_{j}}^{(j)} \frac{\partial^{2} w_{n_{j}}^{(j)}(t)}{\partial t^{2}}, & \text { if } l_{n_{j}}^{(j)}=0 .\end{cases}
$$

where the sum is taken over all the outgoing edges. For an edge $e_{j}$ incident with a pendant vertex (except of the root) we impose the Dirichlet boundary condition:

$$
w_{n_{j}+1}^{(j)}(t)=0
$$

At the root we will consider the Dirichlet condition

$$
\mathbf{w}(t)=0 \text {, i.e. } w_{0}^{(j)}(t)=0
$$

for all $j$ corresponding to the edges incident with the root. We call problem (2.1)-(2.5) the Dirichlet boundary value problem on $T$. 
To obtain the Neumann boundary value problem we change (2.5) for the generalized Neumann conditions

$$
w_{0}^{(j)}(t)=w_{0}^{(l)}(t)
$$

for all $j$ and $l$ corresponding to the edges incident with the root and

$$
\sum_{j=1}^{d(\mathbf{v})} \frac{w_{0}^{(j)}(t)-w_{1}^{(j)}(t)}{l_{0}^{(j)}}=0 .
$$

We exclude from consideration the case with a mass at the root.

If the root is a pendant vertex then we denote by $e_{1}$ the edge incident with the root. In this case the Dirichlet condition (2.5) at the root is

$$
\mathbf{w}(t)=0 \text {, i.e. } w_{0}^{(1)}(t)=0
$$

and the Neumann conditions (2.6), (2.7) in this case can be reduced to

$$
\mathbf{w}(t)=w_{0}^{(1)}(t)=w_{1}^{(1)}(t) .
$$

Substituting $w_{j}^{(k)}(t)=e^{i \lambda t} u_{j}^{(k)}, w_{i}(t)=e^{i \lambda t} u_{i}$ and $\mathbf{w}(t)=e^{i \lambda t} \mathbf{u}$ into (2.1)(2.9) we obtain the corresponding spectral problems:

Dirichlet problem. For each edge:

$$
\begin{gathered}
\frac{u_{k}^{(j)}-u_{k+1}^{(j)}}{l_{k}^{(j)}}+\frac{u_{k}^{(j)}-u_{k-1}^{(j)}}{l_{k-1}^{(j)}}-m_{k}^{(j)} \lambda^{2} u_{k}^{(j)}=0, \\
\left(k=1,2, \ldots, \tilde{n}_{j}, j=1,2, \ldots, q\right) .
\end{gathered}
$$

For each interior vertex (except for the root) with incoming edge $e_{j}$ and outgoing edges $e_{r}$ we have

$$
u_{\tilde{n}_{j}+1}^{(j)}=u_{0}^{(r)}
$$

and

$$
\frac{u_{\tilde{n}_{j}+1}^{(j)}-u_{\tilde{n}_{j}}^{(j)}}{l_{\tilde{n}_{j}}^{(j)}}+\sum_{r} \frac{u_{0}^{(r)}-u_{1}^{(r)}}{l_{0}^{(r)}}= \begin{cases}0, & \text { if } l_{n_{j}}^{(j)}>0 \\ -m_{n_{j}}^{(j)} \lambda^{2} u_{n_{j}}^{(j)}, & \text { if } l_{n_{j}}^{(j)}=0 .\end{cases}
$$

For an edge $e_{j}$ incident with a pendant vertex (except of the root) we have the Dirichlet boundary condition:

$$
u_{n_{j}+1}^{(j)}=0
$$

At the root we have the Dirichlet condition

$$
\mathbf{u}=0 \text {, i.e. } u_{0}^{(j)}=0
$$

for all $j$ corresponding to the edges incident with the root. 
If the root is a pendant vertex and $e_{1}$ the edge incident with the root then instead of (2.14) at the root we have

$$
u_{0}^{(1)}=0 .
$$

Neumann problem. The Neumann problem on $T$ consists of equations (2.10)-(2.13), of

$$
u_{0}^{(j)}=u_{0}^{(l)}
$$

for all $j$ and $l$ corresponding to the edges incident with the root and of

$$
\sum_{j=1}^{d(\mathbf{v})} \frac{u_{0}^{(j)}-u_{1}^{(j)}}{l_{0}^{(j)}}=0 .
$$

If the root is a pendant vertex and $e_{1}$ the edge incident with the root then instead of (2.16) and (2.17) at the root we have

$$
u_{0}^{(1)}=u_{1}^{(1)} \text {. }
$$

\section{Auxiliary Results}

Here we give some lemmas which are used in Section 4 .

Definition 3.1. A rational function $f(z)$ is said to be a Nevanlinna function if:

(i) it is analytic in the half-planes $\operatorname{Im} z>0$ and $\operatorname{Im} z<0$;

(ii) $f(\bar{z})=\overline{f(z)}(\operatorname{Im} z \neq 0)$;

(iii) $\operatorname{Im} z \operatorname{Im} f(z) \geqslant 0$ for $\operatorname{Im} z \neq 0$.

Definition 3.2. A rational Nevanlinna function $f(z)$ is said to be an $S$ function if $f(z)>0$ for $z<0$.

Definition 3.3. A rational $S$-function $f(z)$ is said to be an $S_{0}$-function if 0 is not a pole of $f(z)$.

The following lemmas are obvious.

Lemma 3.4. Suppose that $f$ and $g$ are rational $S_{0}$-functions, then $f+g$ and $\left(f^{-1}+g^{-1}\right)^{-1}$ are also $S_{0}$-functions.

Lemma 3.5. Let $\phi_{j}$ be a rational $S_{0}$ function with $n_{j}$ zeros and $n_{j}$ poles for $j=1,2, \ldots, s$. Then

$$
\frac{1}{\sum_{j=1}^{s} \frac{1}{\phi_{j}}}
$$


is a rational $S_{0}$ function with $\tilde{n}$ zeros and $\tilde{n}$ poles where

$$
\tilde{n} \geqslant \max \left\{n_{1}, n_{2}, \ldots, n_{s}\right\} .
$$

Lemma 3.6. (see [14, Lemma 2.2]) Let

$$
\varphi=a_{0}+\frac{1}{-b_{1} z+\frac{1}{a_{1}+\frac{1}{-b_{2} z+\ldots+\frac{1}{a_{r-1}+\frac{1}{-b_{r} z+\frac{1}{a_{r}+\phi}}}}} .}
$$

where $a_{j}>0$ for $j=0,1, \ldots, r-1, a_{r} \geqslant 0, b_{j}>0$ for $j=1,2, \ldots, r$ and let $\phi$ be a rational $S_{0}$-function with $\hat{n}$ poles and $\hat{n}$ zeros. Then $\varphi$ is a rational $S_{0}$-function with $\hat{n}+r$ zeros and $\hat{n}+r$ poles.

Lemma 3.7. A rational function

$$
f(z)=C \prod_{k=1}^{n} \frac{1-\frac{z}{\left(\nu_{k}\right)^{2}}}{1-\frac{z}{\left(\mu_{k}\right)^{2}}}
$$

is an $S_{0}$-function if and only if $C>0$ and

$$
0<\left(\mu_{1}\right)^{2}<\left(\nu_{1}\right)^{2}<\ldots<\left(\mu_{n}\right)^{2}<\left(\nu_{n}\right)^{2} .
$$

Lemma 3.8. (see e.g. [10, Chapter II.2, p.19/26]). A rational function

$$
f(z)=C \prod_{k=1}^{n} \frac{1-\frac{z}{\left(\nu_{k}\right)^{2}}}{1-\frac{z}{\left(\mu_{k}\right)^{2}}}
$$

is an $S_{0}$-function if and only if $0<\left(\nu_{1}\right)^{2}<\left(\nu_{2}\right)^{2}<\ldots<\left(\nu_{k}\right)^{2}$ and

$$
f(z)^{-1}=\sum_{k=1}^{n} \frac{A_{k}}{z-\left(\nu_{k}\right)^{2}}+B
$$

where $A_{k}>0$ for $k=1,2, \ldots, n$ and

$$
B>\sum_{k=1}^{n} \frac{A_{k}}{\left(\nu_{k}\right)^{2}} \text {. }
$$




\section{Direct PRoblem}

Here and in the sequel we consider a tree $T$ rooted at a pendant vertex. The Dirichlet problem on this tree consists of equations (2.10)-(2.13) and (2.15), while the Neumann problem on it consists of (2.10)-(2.13) and (2.18).

First of all we notice that interior vertices of degree 2 do not influence the results and we can assume absence of such vertices without losses of generality. Let $P$ be a path in the tree $T$ involving the maximum number of masses. Obviously it starts and finishes with pendant vertices. We denote the initial vertex by $v_{0}$ and choose it as the root of the tree. The enumeration of other vertices is arbitrary. We direct the edges away from the root. Denote by $e_{i}$ the edge incoming into a vertex $v_{i}$ for all $i$. Then

$$
P: v_{0} \rightarrow v_{1} \rightarrow v_{s_{2}} \rightarrow v_{s_{3}} \rightarrow \ldots \rightarrow v_{s_{r-1}} \rightarrow v_{s_{r}} .
$$

Here $r$ is a combinatorial length of the path. Deleting $v_{0}$ and $e_{1}$ we obtain a new tree $T^{\prime}$ rooted at the vertex $v_{1}$.

Since $d\left(v_{1}\right)>2$ we can divide our tree $T^{\prime}$ into subtrees

$$
T_{1}^{\prime}, T_{2}^{\prime}, \ldots, T_{d\left(v_{1}\right)-1}^{\prime}
$$

having $v_{1}$ as the only common vertex. (We say that $T_{1}^{\prime}, T_{2}^{\prime}, \ldots, T_{d\left(v_{1}\right)-1}^{\prime}$ are complementary subtrees of $T^{\prime}$ (see Fig. 4.1).

Denote by $\phi_{N}(\mathbf{v})$ the characteristic polynomial of problem (2.10)-(2.13), (2.18) on the tree $T$ and by $\phi_{D}(\mathbf{v})$ the characteristic polynomial of problem (2.10)-(2.13), (2.15) on this tree. These polynomials are normalized such that

$$
\frac{\phi_{D(v)}(0)}{\phi_{N(v)}(0)}=l_{1}+\frac{1}{\sum_{r=1}^{d\left(v_{1}\right)-1} \frac{\phi_{N_{r}\left(v_{1}\right)}(0)}{\phi_{D_{r}\left(v_{1}\right)}(0)}},
$$

$\phi_{D, r\left(v_{1}\right)}(z)$ is the characteristic polynomial of the Dirichlet problem (2.10)(2.13), (2.15) on $T_{r}^{\prime}$ and $\phi_{N, r\left(v_{1}\right)}(z)$ is the characteristic polynomial of the Neumann (2.10)-(2.13), (2.18) on $T_{r}^{\prime}$ and this expansion can be continued.

The following result was proved in [12] (see the proof of Corollary 2.9 there). In that paper it was assumed absence of point masses at the interior vertices of the tree $\left(l_{n_{j}}^{(j)}>0\right.$ for all $\left.j\right)$ but the proof remains true if $l_{n_{j}}^{(j)}=0$ for some values of $j$. 


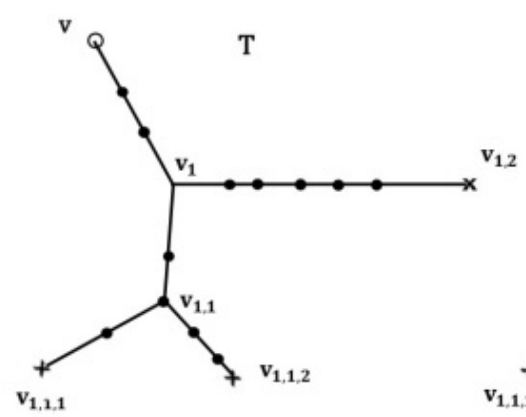

$\mathbf{T}^{\prime}$
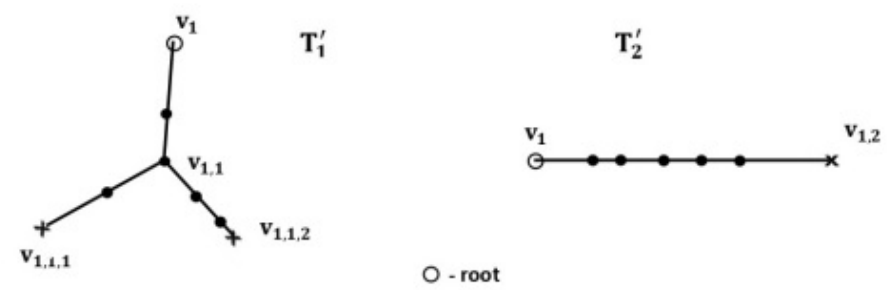

Figure 4.1.

Theorem 4.1. Let the root $\mathbf{v}$ be a pendant vertex of a tree $T$. Then the quotient $\frac{\phi_{D(\mathbf{v})}(z)}{\phi_{N(\mathbf{v})}(z)}$ can be expanded in continued fraction

$$
\frac{\phi_{D(\mathbf{v})}(z)}{\phi_{N(\mathbf{v})}(z)}=l_{0}^{(1)}+\frac{1}{-m_{1}^{(1)} z+\frac{1}{l_{1}^{(1)}+\frac{1}{-m_{2}^{(1)} z+\ldots+\frac{1}{-m_{n_{1}}^{(1)} z+\frac{1}{l_{n_{1}}^{(1)}+\frac{\phi_{D\left(v_{1}\right)}(z)}{\phi_{N\left(v_{1}\right)}(z)}}}}}}
$$

where $\phi_{D\left(v_{1}\right)}(z)$ is the characteristic polynomial of problem (2.10)-(2.14) on $T^{\prime}$ and $\phi_{N\left(v_{1}\right)}(z)$ is the characteristic polynomial of problem (2.10)-(2.13), (2.16), (2.17) on $T^{\prime}$,

$$
\frac{\phi_{D\left(v_{1}\right)}(z)}{\phi_{N\left(v_{1}\right)}(z)}=\frac{1}{\sum_{r=1}^{d\left(v_{1}\right)-1} \frac{\phi_{N_{r}\left(v_{1}\right)}(z)}{\phi_{D_{r}\left(v_{1}\right)}(z)}}
$$

where $d\left(v_{1}\right)$ is the degree of $v_{1}$ as a vertex of $T, \phi_{D_{r}\left(v_{1}\right)}$ is the characteristic polynomial of problem (2.10)-(2.13), (2.15) on $T_{r}$ and $\phi_{N_{r}\left(v_{1}\right)}$ is the 
characteristic polynomial of problem (2.10)-(2.13), (2.18) on it.

$$
\frac{\phi_{D_{r}\left(v_{1}\right)}(z)}{\phi_{N_{r}\left(v_{1}\right)}(z)}=l_{0}^{(r)}+\frac{1}{-m_{1}^{(r)} z+\frac{1}{l_{1}^{(r)}+\frac{1}{-m_{2}^{(r)} z+\ldots+\frac{1}{-m_{n_{r}}^{(r)} z+\frac{1}{l_{n_{r}}^{(r)}+f_{r}(z)}}}}}
$$

In turn each $f_{r}$ can be expanded similarly to (4.2), (4.3).

Remark 4.2. It is proved in [13] that the number of distinct eigenvalues of each of the problems (2.10)-(2.13), (2.15) and (2.10)-(2.13), (2.18) on a tree bearing masses on every edge is not less than the maximum number of point masses on a path in this tree.

\section{INVERSE PROBLEM}

Theorem 5.1. Suppose $\left\{\mu_{k}\right\}_{k=-n, k \neq 0}^{n}$ and $\left\{\nu_{k}\right\}_{k=-n, k \neq 0}^{n}$ are symmetric $\left(\mu_{-k}=-\mu_{k}, \nu_{-k}=-\nu_{k}\right)$ and monotonic sequences of real numbers which interlace:

$$
0<\left(\mu_{1}\right)^{2}<\left(\nu_{1}\right)^{2}<\ldots<\left(\mu_{n}\right)^{2}<\left(\nu_{n}\right)^{2}
$$

Let $T$ be a metric tree of a prescribed formrooted at a pendant vertex $\mathbf{v}$ with prescribed lengths of edges $l_{j}>0(j=1,2, \ldots, q, q$ is the number of edges in T). Then

1) there exist numbers $n_{j} \in\{0\} \cup \mathbb{N}(j=1,2, \ldots, q)$, sequences of positive numbers $\left\{m_{k}^{(j)}\right\}_{k=1}^{n_{j}}$ (point masses on the edge $e_{j}, j=1,2, \ldots, q$ ) and numbers $\left\{l_{k}^{(j)}\right\}_{k=0}^{n_{j}}\left(l_{k}^{(j)}>0\right.$ for all $k=0,1, \ldots, n_{j}-1, l_{n_{j}} \geqslant 0$ for all $j=1,2, \ldots, g$ such that $\sum_{k=0}^{n_{j}} l_{k}^{(j)}=l_{j}, \sum_{j=1}^{q} n_{j}=n$, the spectrum of Neumann problem (2.10)-(2.13), (2.18), coincides with $\left\{\mu_{k}\right\}_{k=-n, k \neq 0}^{n}$ and the spectrum of Dirichlet problem (2.10)-(2.13), (2.15) coincides with $\left\{\nu_{k}\right\}_{k=-n, k \neq 0}^{n}$;

2) the two spectra $\left\{\mu_{k}\right\}_{k=-n, k \neq 0}^{n}$ and $\left\{\nu_{k}\right\}_{k=-n, k \neq 0}^{n}$ and the length $l_{1}$ of the edge incident with the root uniquely determine the masses $\left\{m_{k}^{(1)}\right\}_{k=1}^{n_{1}}$ (point masses on the edge $e_{1}$ ) and lengths $\left\{l_{k}^{(1)}\right\}_{k=0}^{n_{1}}$ of the subintervals on this edge. 
Proof. First of all we consider the rational function

$$
F(z):=\Phi_{T, \mathbf{v}} \prod_{k=1}^{n} \frac{1-\frac{z}{\left(\mu_{k}\right)^{2}}}{1-\frac{z}{\left(\nu_{k}\right)^{2}}}
$$

where $\Phi_{T, \mathbf{v}}>0$ is the form characteristic of the tree $T$ which depends only on the form of the tree and the lengths of the edges. It can be found substituting $z=0$ in (4.1)-(4.3): $\Phi_{T, \mathbf{v}}:=\frac{\phi_{N(\mathbf{v})}(0)}{\phi_{D(\mathbf{v})}(0)}$.

Let $e_{1}$ be the edge connecting $\mathbf{v}$ with $v_{1}$ and let $l_{1}$ be the length of this edge. Substituting $z=0$ in (4.1) we obtain

$$
\Phi_{T, \mathbf{v}}^{-1}=l_{1}+\frac{\phi_{D\left(v_{1}\right)}(0)}{\phi_{N\left(v_{1}\right)}(0)}>l_{1}
$$

Due to (5.1) and $\Phi_{T, \mathbf{v}}>0, F^{-1}(z)$ is an $S_{0}$-function and, therefore can be presented as

$$
F(z)^{-1}=a_{0}+\frac{1}{-b_{1} z+\frac{1}{a_{1}+\frac{1}{-b_{2} z+\ldots+\frac{1}{a_{n-1}+\frac{1}{-b_{n} z+\frac{1}{a_{n}}}}}},}
$$

where $a_{k}>0$ for $k=0,1, \ldots, n$ and $b_{k}>0$ for $k=1,2, \ldots, n$.

Since $F^{-1}(0)=\sum_{k=0}^{n} a_{k}=\Phi_{T, \mathbf{v}}^{-1} \geqslant l_{1}$, we can choose the integer number $n_{1}$ such that

$$
\sum_{k=0}^{n_{1}-1} a_{k} \leqslant l_{1}<\sum_{k=0}^{n_{1}} a_{k}
$$

and present $F(z)^{-1}$ as follows:

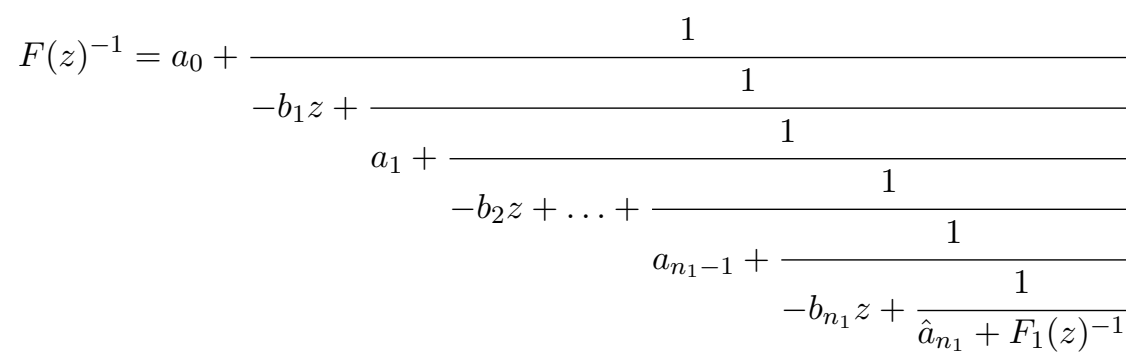


where

$$
\hat{a}_{n_{1}}=l_{1}-\sum_{k=0}^{n_{1}-1} a_{k}
$$

and

$$
F_{1}(z)^{-1}=a_{n_{1}}-\hat{a}_{n_{1}}+\frac{1}{-b_{n_{1}+1} z+\frac{1}{a_{n_{1}+1}+\frac{1}{-b_{n_{1}+2} z+\ldots+\frac{1}{a_{n-1}+\frac{1}{-b_{n} z+\frac{1}{a_{n}}}}}} .}
$$

We identify $\left\{a_{0}, a_{1}, \ldots, a_{n_{1}-1}, \hat{a}_{n_{1}}\right\}$ with the subintervals of the edge $e_{1}$ and $\left\{b_{1}, b_{2}, \ldots, b_{n_{1}}\right\}$ with the masses on it:

$$
\begin{aligned}
a_{k} & =l_{k}^{(1)},\left(k=0,1, \ldots, n_{1}-1\right), \\
\hat{a}_{n_{1}} & =l_{n_{1}}^{(1)}, \\
b_{k} & =m_{k}^{(1)},\left(k=1,2, \ldots, n_{1}\right) .
\end{aligned}
$$

Since $d\left(v_{1}\right)>2$ in $T$, we divide the tree $T^{\prime}$ into $d\left(v_{1}\right)-1$ complementary subtrees $T_{j}$ rooted at $v_{1}$ each (see Fig. 5.1). It is clear that $F_{1}(z)^{-1}$ belongs to $S_{0}$ and therefore

where

$$
F_{1}(z)=\Phi_{T^{\prime}, v_{1}} \prod_{k=1}^{n-n_{1}} \frac{1-\frac{z}{\left(\tilde{\mu}_{k}\right)^{2}}}{1-\frac{z}{\left(\tilde{\nu}_{k}\right)^{2}}}
$$

$$
\begin{gathered}
\Phi_{T^{\prime}, v_{1}}^{-1}=\Phi_{T, \mathbf{v}}^{-1}-l_{1}, \\
0<\left(\tilde{\mu}_{1}\right)^{2}<\left(\tilde{\nu}_{1}\right)^{2}<\left(\tilde{\mu}_{2}\right)^{2}<\ldots<\left(\tilde{\nu}_{n-n_{1}}\right)^{2}
\end{gathered}
$$

It is known that if $F_{1}(z)^{-1}$ belongs to $S_{0}$ then

$$
\begin{gathered}
F_{1}(z)=\sum_{k=1}^{n-n_{1}} \frac{A_{k}}{z-\left(\tilde{\nu}_{k}\right)^{2}}+B, \\
A_{k}>0, B>0
\end{gathered}
$$

and since $F^{-1}(0)=\Phi_{T^{\prime}, v_{1}}^{-1}$ we have

$$
B=\Phi_{T^{\prime}, v_{1}}+\sum_{k=1}^{n-n_{1}} \frac{A_{k}}{\left(\tilde{\nu}_{k}\right)^{2}} .
$$



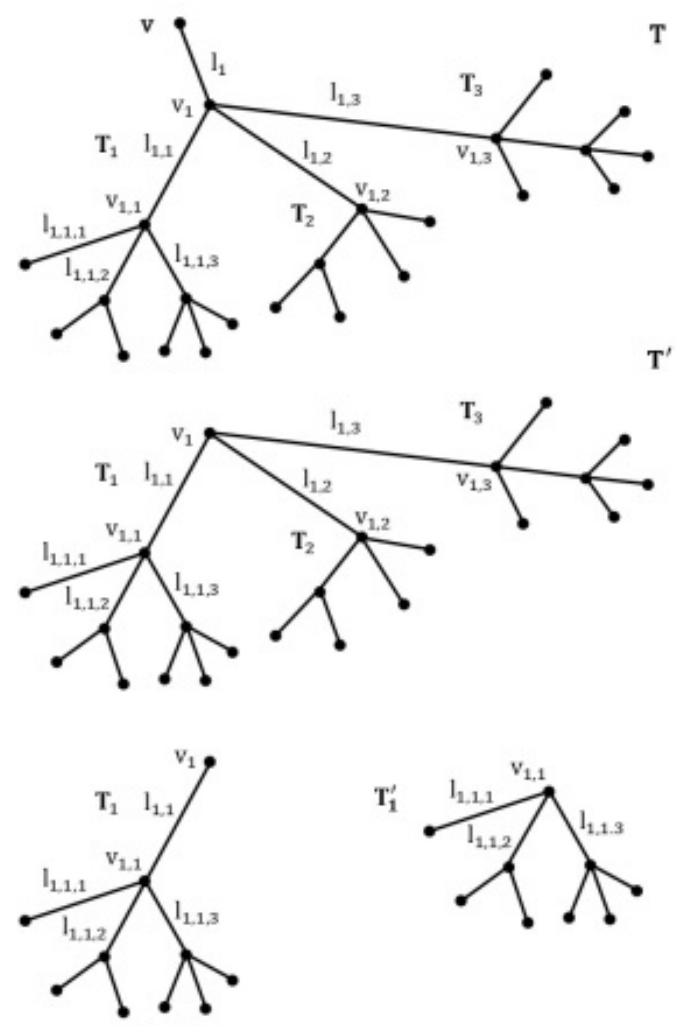

FiguRE 5.1.

Let us choose nonnegative integers $N_{j}\left(j=1,2, \ldots, d\left(v_{1}\right)-1\right)$ such that

$$
\sum_{j=1}^{d\left(v_{1}\right)-1} N_{j}=n-n_{1} .
$$

Since $\Phi_{T^{\prime}, v_{1}}>0$ and therefore,

$$
B>\sum_{k=1}^{n-n_{1}} \frac{A_{k}}{\left(\tilde{\nu}_{k}\right)^{2}}
$$

We arrange the set $\left\{\left(\tilde{\nu}_{k}\right)^{2}\right\}_{k=1}^{n-n_{1}}$ as the union of disjoint sets

$$
\left\{\left(\tilde{\nu}_{k_{s}^{(1)}}\right)^{2}\right\}_{s=1}^{N_{1}}, \quad\left\{\left(\tilde{\nu}_{k_{s}^{(2)}}\right)^{2}\right\}_{s=1}^{N_{2}}, \quad \ldots, \quad\left\{\left(\tilde{\nu}_{k_{s}^{(d(v)-1)}}\right)^{2}\right\}_{s=1}^{N_{d\left(v_{1}\right)-1}}
$$


and choose numbers $B_{j}$

$\left(j=1,2, \ldots, d\left(v_{1}\right)-1\right)$ such that

$$
\sum_{j=1}^{d\left(v_{1}\right)-1} B_{j}=B
$$

and

$$
B_{j}>\sum_{s=1}^{N_{j}} \frac{A_{k_{s}}}{\left(\tilde{\nu}_{k_{s}}^{(j)}\right)^{2}}
$$

Then

$$
F_{1}(z)=\Phi_{T^{\prime}, v_{1}} \prod_{k=1}^{n-n_{1}} \frac{1-\frac{z}{\left(\tilde{\mu}_{k}\right)^{2}}}{1-\frac{z}{\left(\tilde{\nu}_{k}\right)^{2}}}=\sum_{j=1}^{d\left(v_{1}\right)-1}\left(\sum_{s=1}^{N_{j}} \frac{A_{k_{s}}}{z-\left(\tilde{\nu}_{k_{s}}^{(j)}\right)^{2}} k_{s}+B_{j}\right)
$$

Put

$$
\Phi_{T_{j}, v_{1}}=B_{j}-\sum_{s=1}^{N_{j}} \frac{A_{k_{s}}}{\left(\tilde{\nu}_{k_{s}}^{(j)}\right)^{2}}>0
$$

We will show that there exists a distribution of masses on the complimentary subtrees $T_{j}\left(j=1,2, \ldots, d\left(v_{1}\right)-1\right), \underset{j=1}{d\left(v_{1}\right)-1} T_{j}=T^{\prime}$ such that

1) the number of masses in $T_{j}$ is $N_{j}$;

2) the form characteristic of $T_{j}$ is $\Phi_{T_{j}, v_{1}}$ and the rational function

$$
\sum_{s=1}^{N_{j}} \frac{A_{k_{s}}}{z-\left(\tilde{\nu}_{k_{s}}^{(j)}\right)^{2}}+B_{j}
$$

having $N_{j}$ simple zeros and $N_{j}$ simple poles is $\frac{\phi_{N}^{(j)}(z)}{\phi_{D}^{(j)}(z)}$, where $\phi_{N}^{(j)}(z)$ and $\phi_{D}^{(j)}(z)$ are the characteristic polynomials for the Neumann and the Dirichlet problems on the subtree $T_{j}$. 
Let us expand $\left(\sum_{s=1}^{N_{j}} \frac{A_{k_{s}}}{z-\left(\tilde{\nu}_{k_{s}}^{(j)}\right)^{2}}+B_{j}\right)^{-1}$ into continued fractions

$$
\begin{aligned}
& \left(\sum_{s=1}^{N_{j}} \frac{A_{k_{s}}}{z-\left(\tilde{\nu}_{k_{s}}^{(j)}\right)^{2}}+B_{j}\right)^{-1}=
\end{aligned}
$$

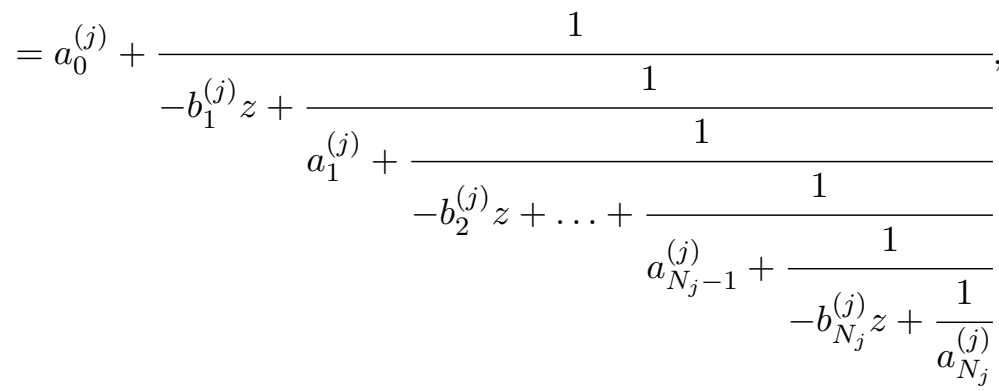

It is clear that due to (5.11) and (5.14) the left hand side of (5.18) is an $S_{0}$-function.

We define $\tilde{n}_{j}$ by the inequalities $\sum_{k=0}^{\tilde{n}_{j}-1} a_{k}^{(j)} \leqslant l_{1, j}<\sum_{k=0}^{\tilde{n}_{j}} a_{k}^{(j)}$ where $l_{1, j}$ is the length of the edge of $T_{j}$ incident with $v_{1}$. Then we can rewrite $(5.18)$ as

$$
\begin{aligned}
& \left(\sum_{s=1}^{N_{j}} \frac{A_{k_{s}}}{z-\left(\tilde{\nu}_{k_{s}}^{(j)}\right)^{2}}+B_{j}\right)^{-1}= \\
& =a_{0}^{(j)}+\frac{1}{-b_{1}^{(j)} z+\frac{1}{a_{1}^{(j)}+\frac{1}{-b_{2}^{(j)} z+\ldots+\frac{1}{a_{\tilde{n}_{j}-1}^{(j)}+\frac{1}{-b_{\tilde{n}_{j}}^{(j)} z+\frac{1}{\tilde{a}_{\tilde{n}_{j}}^{(j)}+F_{j, 1}^{-1}(z)}}}}},}
\end{aligned}
$$

where

$$
\tilde{a}_{\tilde{n}_{j}}^{(j)}=l_{1, j}-\sum_{k=0}^{\tilde{n}_{j}-1} a_{k}^{(j)}>0
$$


and

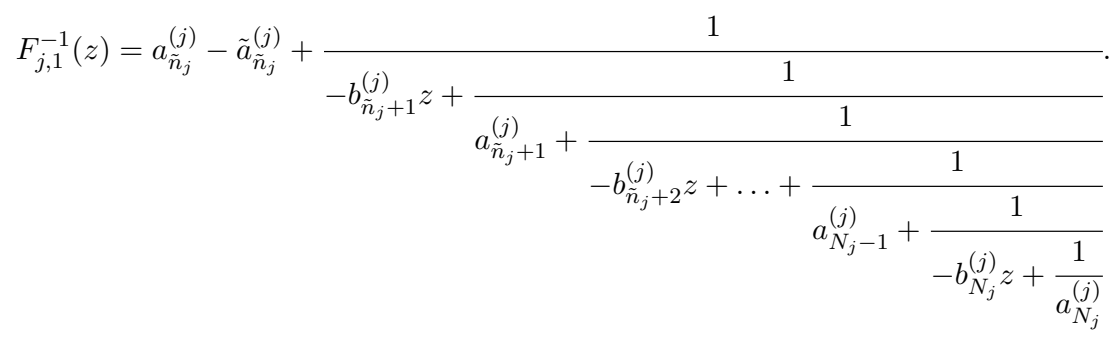

We identify numbers $a_{0}^{(j)}, a_{1}^{(j)}, \ldots, a_{\tilde{n}_{j}-1}^{(j)}, \tilde{a}_{\tilde{n}_{j}}^{(j)}$ with the lengths of subintervals $l_{0}^{(1, j)}, l_{1}^{(1, j)}, \ldots, l_{\tilde{n}_{j}}^{(1, j)}$ and $b_{1}^{(j)}, b_{2}^{(j)}, \ldots, b_{\tilde{n}_{j}}^{(j)}$ with the values of masses $m_{1}^{(1, j)}, \ldots, m_{\tilde{n}_{j}}^{(1, j)}$ on the edge $e_{1, j}$. In the same way as (5.9) we obtain

$$
F_{j, 1}(z)=\sum_{k=1}^{N_{j}-\tilde{n}_{j}} \frac{\tilde{A}_{k}}{z-\left(\tilde{\nu}_{k}\right)^{2}}+\tilde{B}_{j}
$$

with $\tilde{A}_{k}^{(j)}>0$ and $\tilde{B}_{j}>\sum_{k=1}^{N_{j}-\tilde{n}_{j}} \frac{\tilde{A}_{k}}{\left(\tilde{\nu}_{k}^{(j)}\right)^{2}}$.

Denote by $v_{1, j}$ the second vertex incident with $e_{1, j}$ and by $d\left(v_{1, j}\right)$ the degree of $v_{1, j}$ Now we consider the tree $T_{j}^{\prime}$ obtained by deleting the edge $e_{1, j}$ from $T_{j}$ (see Fig. 5.1). Let $v_{1, j}$ be the root of $T_{j}^{\prime}$.

Substituting $z=0$ into (5.15) and making use of (5.16) we obtain

$$
\Phi_{T^{\prime}, v_{1}}=\sum_{i=1}^{d\left(v_{1}\right)-1} \Phi_{T_{j}, v_{1}}
$$

On the other hand, (5.19) implies

$$
\Phi_{T_{j}, v_{1}}^{-1}=l_{1, j}+F_{j, 1}(0)^{-1}
$$

and consequently, $F_{j, 1}^{-1}(0)=\Phi_{T_{j}^{\prime}, v_{1}}^{-1}$.

We continue this procedure. Finally we obtain a branching continued fraction. We identify

$$
\begin{aligned}
a_{k}^{(j)} & =l_{k}^{(1, j)},\left(k=0,1, \ldots, n_{j}-1\right), \\
\hat{a}_{n_{j}} & =l_{n_{j}}^{(1, j)} \\
b_{k}^{(j)} & =m_{k}^{(1, j)},\left(k=1,2, \ldots, n_{1, j}\right) .
\end{aligned}
$$


According to Theorem 4.1 the problems (2.10)-(2.13), (2.18) and (2.10)(2.13), (2.15) with these masses and subintervals have the quotient

$$
\frac{\phi_{D(\mathbf{v})}(z)}{\phi_{N(\mathbf{v})}(z)}=F^{-1}(z)
$$

where $F(z)$ is given by (5.2).

Since the equation (5.6) uniquely determines the sets $\left\{a_{k}\right\}_{k=0}^{n_{1}-1} \cup\left\{\hat{a}_{n_{1}}\right\}$ and $\left\{b_{k}\right\}_{k=1}^{n_{1}}$ and (5.7) uniquely determines the number $n_{1}$ Statement 2) is valid.

Theorem 5.2. Suppose $\left\{\mu_{k}\right\}_{k=-n, k \neq 0}^{n}$ and $\left\{\nu_{k}\right\}_{k=-n, k \neq 0}^{n}$ are symmetric $\left(\mu_{-k}=-\mu_{k}, \nu_{-k}=-\nu_{k}\right)$ and monotonic sequences of real numbers which interlace:

$$
0<\left(\mu_{1}\right)^{2}<\left(\nu_{1}\right)^{2}<\ldots<\left(\mu_{n}\right)^{2}<\left(\nu_{n}\right)^{2} .
$$

Let $T$ be a metric tree of a prescribed form rooted at a pendant vertex $\mathbf{v}$ with prescribed number of masses on the edges $n_{j} \geqslant 0(j=1,2, \ldots, q, q$ is the number of edges in $\left.T, n_{j} \geqslant 0, \sum_{j=1}^{q} n_{j}=n\right)$.

Then there exist sequences of positive numbers $\left\{m_{k}^{(j)}\right\}_{k=1}^{n_{j}}$ (point masses on the edge $\left.e_{j}, j=1,2, \ldots, q\right)$ and numbers $\left\{l_{k}^{(j)}\right\}_{k=0}^{n_{j}}\left(l_{k}^{(j)}>0\right.$ for all $k=0,1, \ldots, n_{j}-1, l_{n_{j}} \geqslant 0$ for all $j=1,2, \ldots, g$ such that the numbers of point masses on the edge $e_{j}$ is $n_{j}(j=1,2, \ldots, q)$ and the spectrum of Neumann problem (2.10)-(2.13), (2.18), coincides with $\left\{\mu_{k}\right\}_{k=-n, k \neq 0}^{n}$ and the spectrum of Dirichlet problem (2.10)-(2.13), (2.15) coincides with $\left\{\nu_{k}\right\}_{k=-n, k \neq 0}^{n}$.

Proof. We choose an arbitrary positive number $\Phi_{T, \mathbf{v}}$ and construct the rational function $F(z)$ as in (5.2) and expand $F^{-1}(z)$ into continued fraction (5.4), choose $l_{1}$ such that satisfies (5.5) and define

$$
\Phi_{T^{\prime}, v_{1}}^{-1}:=\Phi_{T, \mathbf{v}}^{-1}-l_{1} .
$$

Since

$$
l_{1}<\sum_{k=0}^{n_{1}} a_{k} \leqslant \sum_{k=0}^{n} a_{k}=F^{-1}(0)=\Phi_{T, \mathbf{v}}^{-1}
$$

we arrive at

$$
\Phi_{T^{\prime}, v_{1}}^{-1}>0
$$

Now $N_{j}\left(j=1,2, \ldots, d\left(v_{1}\right)-1\right)$ which is the total number of point masses on $T_{j}$ can be calculated using the given values of numbers of point masses $n_{j}$ corresponding to the edges of $T_{j}$. 
We arrange the set $\left\{A_{k}\right\}_{k=1}^{n-n_{1}}$ of coefficients in (5.10) into groups $\left\{A_{k_{s}}^{(j)}\right\}_{s=1}^{N_{j}}$ $(j=1,2, \ldots, d(\mathbf{v})-1)$ in arbitrary way and choose positive numbers $B_{j}$ in so that (5.13), (5.14) hold. The latter is possible due to (5.12). Denote by $\Phi_{T_{j}, v_{1}}$ the values obtained via (5.16) and regarded as the form factors of the trees $T_{j}$ we are constructing. Due to $A_{j}>0$ and (5.14) the functions $\left(\sum_{s=1}^{N_{j}} \frac{A_{k_{s}}}{z-\left(\nu_{k_{s}}^{(j)}\right)^{2}}+B_{j}\right)^{-1}$ are $S_{0}$-functions and, consequently, (5.19) holds. Let us choose the length $l_{j}$ of the edge $e_{j}$ incident with $\mathbf{v}$ so that

$$
\sum_{k=0}^{n_{j}-1} a_{k}^{(j)} \leqslant l_{j}<\sum_{k=0}^{n_{j}} a_{k}^{(j)}
$$

where $n_{j}$ is the number of the masses on $e_{j}$. Then we present the functions as in (5.19) and (5.20) but with given $n_{j}$ instead of $\tilde{n}_{j}$. Then we continue this procedure as in the proof of Theorem 5.1.

\section{REFERENCES}

[1] P.F. Kurchanov A.M. Filimonov, A.D. Myshkis. Some unexpected results in the classical problem of vibrations of the string with $n$ beads when $n$ is large. C.R. Acad. Sci. Paris, Ser. I. Math., 313(6):961-965, 1991.

[2] O. Boyko, V. Pivovarchik. Inverse spectral problem for a star graph of Stieltjes strings. Methods Funct. Anal. Topology, 14(2):159-167, 2008.

[3] Wilhelm Cauer. Die verwirklichung von wechselstromwiderständen vorgeschriebener frequenzabhängigkeit. Archiv für Elektrotechnik, 17(4):355-388, April 1926, doi: $10.1007 /$ bf01662000.

[4] A. M. Filimonov, A. D. Myshkis. On properties of large wave effect in classical problem of bead string vibration. J. Difference Equ. Appl., 10(13-15):1171-1175, 2004, doi: $10.1080 / 10236190410001652757$.

[5] F. P. Gantmacher, M. G. Krein. Oscillation matrices and kernels and small vibrations of mechanical systems. AMS Chelsea Publishing, Providence, RI, revised edition, 2002, doi: $10.1090 / \mathrm{chel} / 345$. Translation based on the 1941 Russian original, Edited and with a preface by Alex Eremenko.

[6] Joseph Genin, John S. Maybee. Mechanical vibration trees. J. Math. Anal. Appl., 45:746-763, 1974, doi: 10.1016/0022-247X(74)90065-1.

[7] Graham M. L. Gladwell. Inverse problems in vibration, volume 119 of Solid Mechanics and its Applications. Kluwer Academic Publishers, Dordrecht, second edition, 2004.

[8] Graham M. L. Gladwell, Antonino Morassi, editors. Dynamical inverse problems: theory and application, volume 529 of CISM International Centre for Mechanical Sciences. Courses and Lectures. SpringerWienNewYork, Vienna, 2011, doi: 10.1007/9783-7091-0696-9. Papers from the CISM Course held in Udine, May 25-29, 2009.

[9] Ernst A. Guillemin. Synthesis of passive networks. Theory and methods appropriate to the realization and approximation problems. John wiley and Sons, Inc., New York; Chapman and Hall, Ltd., London, 1958.

[10] W.F. Donoghue Jr. Monotone matrix functions and analytic continuation. Springer Verlag, New York, 1974. 
[11] V. A. Marchenko. Introduction to the theory of inverse problems of spectral analysis. Acta, Kharkov, 2005 (in Russian).

[12] V. Pivovarchik. Existence of a tree of Stieltjes strings corresponding to two given spectra. J. Phys. A, 42(37):375213, 16, 2009, doi: 10.1088/1751-8113/42/37/375213.

[13] V. M. Pivovarchik. On the minimum number of distinct eigenvalues in a problem on a tree of Stieltjes strings. Ukraïn. Mat. Zh., 72(1):135-141, 2020.

[14] Vyacheslav Pivovarchik, Natalia Rozhenko, Christiane Tretter. Dirichlet-Neumann inverse spectral problem for a star graph of Stieltjes strings. Linear Algebra Appl., 439(8):2263-2292, 2013, doi: 10.1016/j.laa.2013.07.003.

[15] Vyacheslav Pivovarchik, Christiane Tretter. Location and multiplicities of eigenvalues for a star graph of Stieltjes strings. J. Difference Equ. Appl., 21(5):383-402, 2015, doi: 10.1080/10236198.2014.992425.

[16] M. Embree S.J. Cox, J.M. Hokanson. One can hear the composition of a string: experiments with an inverse eigenvalue problem. SIAM Rev., 54(1):157-178, 2012.

[17] Che-Wei Tsao, Chun-Kong Law. The Stieltjes string and its associated nodal points. Oper. Matrices, 13(2):363-373, 2019, doi: 10.7153/oam-2019-13-26.

[18] Lu Yang, Yongxia Guo, Guangsheng Wei. Inverse problem for a Stieltjes string damped at an interior point. Integral Equations Operator Theory, 92(4):Paper No. 30, 16, 2020, doi: $10.1007 / \mathrm{s} 00020-020-02587-4$.

[19] Lu Yang, Guangsheng Wei, Vyacheslav Pivovarchik. Direct and inverse spectral problems for a star graph of Stieltjes strings damped at a pendant vertex. Inverse Probl. Imaging, 15(2):257-270, 2021, doi: 10.3934/ipi.2020063.

Received: September 10, 2020, accepted: October, 12, 2020.

Anastasia Dudko

South Ukrainian National Pedagogical University named after K. D. Ushynsky

Email: nastysha00301@gmail.com

ORCID: orcid.org/0000-0002-0722-9751

Vyacheslav Pivovarchik

South Ukrainian National Pedagogical University named after K. D. Ushynsky

Email: vpivovarchik@gmail.com

ORCID: orcid.org/0000-0001-7400-7270 